\title{
The Chandra view of the 3C/FR I sample of low luminosity radio-galaxies
}

\author{
B. Balmaverde ${ }^{1}$, A. Capetti ${ }^{2}$, and P. Grandi ${ }^{3}$ \\ 1 Dipartimento di Fisica Generale, Universitá di Torino, via P. Giuria 1, 10125 Torino, Italy \\ e-mail: balmaverde@ph. unito.it \\ 2 INAF - Osservatorio Astronomico di Torino, via Osservatorio 20, 10025 Pino Torinese, Italy \\ e-mail: capetti@to.astro.it \\ 3 INAF - Istituto di Astrofisica Spaziale e Fisica Cosmica, via Gobetti 101, 40129 Bologna, Italy \\ e-mail: grandi@bo.iasf.cnr.it
}

Received 8 July 2005 / Accepted 19 December 2005

\begin{abstract}
We present results from Chandra observations of the 3C/FR I sample of low luminosity radio-galaxies. We detected a power-law nuclear component in 12 objects out of the 18 with available data. In 4 galaxies we detected nuclear X-ray absorption at a level of $N_{\mathrm{H}} \sim(0.2-6) \times$ $10^{22} \mathrm{~cm}^{-2}$. X-ray absorbed sources are associated with the presence of highly inclined dusty disks (or dust filaments projected onto the nuclei) seen in the HST images. This suggests the existence of a flattened X-ray absorber, but of much lower optical depth than in classical obscuring tori. We thus have an unobstructed view toward most FR I nuclei, while absorption plays only a marginal role in the remaining objects.

Three pieces of evidence support a jet origin for the X-ray cores: i) the presence of strong correlations between the nuclear luminosities in the radio, optical, and X-ray bands, extending over 4 orders of magnitude and having a much smaller dispersion ( $\sim 0.3$ dex) when compared to similar trends found for other classes of AGNs, all of which points to a common origin for the emission in the three bands; ii) the close similarity of the broad-band spectral indices with the sub-class of BL Lac objects sharing the same range of extended radio-luminosity, in accord with the FR I/BL Lacs unified model; iii) the presence of a common luminosity evolution of spectral indices in both FR I and BL Lacs. The low luminosities of the X-ray nuclei, regardless of their origin, strengthens the interpretation of low efficiency accretion in low luminosity radio-galaxies.
\end{abstract}

Key words. galaxies: active - galaxies: BL Lacertae objects: general - galaxies: nuclei - galaxies: jets

\section{Introduction}

By exploring the properties of low-luminosity radio-galaxies (LLRG), it is possible to extend the study of active galactic nuclei (AGN) and, in particular, of the radio-loud sub-population, toward the lowest level of nuclear luminosity. This offers the opportunity to improve our understanding of the mechanism of accretion onto super-massive black holes and of their radiative manifestations. In this respect, it is important to note that LLRG not only represent the bulk of radio-loud active galaxies, due to the steepness of their luminosity function, but also a substantial fraction of the overall galaxy population. In fact LLRG are associated to a fraction as high as $40 \%$ of all bright elliptical and lenticular galaxies (Sadler et al. 1989; Auriemma et al. 1977). Thus the presence of a low power radio galaxy represents by far the most common manifestation of nuclear activity in early type galaxies.

Low-luminosity AGN (LLAGN) also represent a link between the high-luminosity AGN and the population of quiescent galaxies, since it is now widely recognized that most (if not all) galaxies host a super-massive black-hole (e.g. Kormendy $\&$ Richstone 1995). The comparison of the different manifestations of nuclear activity across the widest possible range of luminosity is then a crucial step in unveiling the connections (and the diversity) between active and non-active galaxies.

Unfortunately, the study of LLRG has been significantly hampered by the contamination from host galaxy emission that, in most observing bands, dominates the emission from the AGN. To constrain the physical processes at work in these objects, it is clearly necessary to disentangle the AGN and the host's contributions via spectral decomposition or highresolution imaging. In the last few years, thanks in particular to the Hubble Space Telescope (HST), this has become routinely possible.

An example of the insights that can be obtained following this approach comes from the results derived from the analysis of the broad-band HST imaging of a sample of LLRG (Chiaberge et al. 1999). In the majority of the targets, HST images revealed the presence of unresolved optical nuclei. The fluxes and luminosities of these sources show a tight 
correlation with the radio cores, extending over four orders of magnitude. This has been interpreted as being due to a common non-thermal emission process in the radio and optical band, i.e. that we are seeing the optical emission from the base of a relativistic jet. The low luminosity of LLRG optical nuclei and the possible dominance of the emission related to out-flowing material indicate a low level of accretion and/or a low radiative efficiency with respect to classical, more luminous AGN. The high fraction $(\sim 85 \%)$ of objects with detected optical nuclear sources suggests a general lack of obscuring molecular tori, a further distinction with respect to other classes of AGN. The tenuous torus structure - when considered together with the low accretion rate, the low mass of the compact emission line regions $\left(10-10^{3} M_{\odot}\right)$, and the limits to the mass of the Broad Line Region $\left(M_{\mathrm{BLR}}<10^{-2} M_{\odot}\right)$ - indicates that a general paucity of gas in the innermost regions of LLRG emerges as the main characterizing difference from more powerful AGN (Capetti et al. 2005).

The advent of Chandra provides us with the unique opportunity to extend the study of LLRG to the X-ray band since its high spatial resolution enables us to isolate any low-power nuclear source associated to LLRG. Furthermore, with respect to the optical data, the spectral capabilities of Chandra allow us to study the spectral behavior of the X-ray nuclei directly. This can be used to quantify the effects of local absorption, as well as the slope of their high energy emission, so as to better constrain the emission processes at work in these sources, particularly when combined in a multi-wavelength analysis that also uses optical and radio observations. This approach has already been followed by several authors in the past (e.g. Capetti et al. 2000b; Hardcastle \& Worrall 2000; Trussoni et al. 2003; Hardcastle et al. 2003), but clearly, the superior capabilities of Chandra warrant re-exploring this issue in much greater depth.

Here we present results obtained from the analysis of Chandra data for the sample of LLRG drawn from the 3C catalogue of radio-sources (Bennett 1962), more specifically those with a morphological classification as an FR I (Fanaroff \& Riley 1974). Considering LLRG from the 3C sample, those studied by Chiaberge et al. (1999) represent an obvious choice. It is the best-studied sample of radio-loud galaxies in existence with a vast array of ground and spaced-based observations for comparison at essentially all accessible wavelengths. Other samples of LLRG have been considered in the literature, but not surprisingly, the coverage provided by Chandra observations for these samples is severely incomplete. For example, only about $10 \%$ of the B2 sample of LLRG (Colla et al. 1975) has been observed with Chandra (to be compared to the $\sim 55 \%$ for the 3C/FR I sample), and the analysis is further obstructed by the unknown selection biases introduced in the choice of the individual targets. Similarly we preferred to focus on the Chandra data alone, to provide the highest possible level of uniformity in the analysis.

The paper is organised as follows: in Sect. 2 we present the observations and the data reduction that lead to the results described in Sect. 3 and, in particular, to the detection of X-ray nuclei in most FR I. In Sect. 4 we explore the effects of the nuclear X-ray absorption, by also relating it to the presence of dust features seen in the HST images. The origin of the X-ray
Table 1. Log of the observation.

\begin{tabular}{lcccc}
\hline \hline Name & Obs Id & Inst & $\begin{array}{c}\text { Exp time } \\
{[\mathrm{ks}]}\end{array}$ & $\begin{array}{c}\text { Frame time } \\
{[\mathrm{s}]}\end{array}$ \\
\hline 3C 028 & 3233 & ACIS/I & 50.38 & 3.1 \\
3C 031 & 2147 & ACIS/S & 44.98 & 3.2 \\
3C 066B & 828 & ACIS/S & 45.17 & 1.5 \\
3C 075 & 4181 & ACIS/I & 21.78 & 3.2 \\
3C 078 & 3128 & ACIS/S & 5.23 & 3.1 \\
3C 083.1 & 3237 & ACIS/S & 95.14 & 3.1 \\
3C 084 & 3404 & ACIS/S & 5.86 & 0.4 \\
3C 189 & 858 & ACIS/S & 8.26 & 0.8 \\
3C 270 & 834 & ACIS/S & 35.18 & 1.8 \\
3C 272.1 & 803 & ACIS/S & 28.85 & 3.2 \\
3C 274 & 1808 & ACIS/S & 14.17 & 0.4 \\
3C 296 & 3968 & ACIS/S & 50.08 & 3.2 \\
3C 317 & 890 & ACIS/S & 37.23 & 3.2 \\
3C 338 & 497 & ACIS/S & 19.72 & 3.2 \\
& 498 & ACIS/S & 19.16 & 3.2 \\
3C 346 & 3129 & ACIS/S & 46.69 & 0.8 \\
3C 348 & 1625 & ACIS/S & 15.00 & 3.2 \\
3C 438 & 3967 & ACIS/S & 47.9 & 3.1 \\
3C 449 & 4057 & ACIS/S & 29.56 & 3.2 \\
\hline
\end{tabular}

nuclei found in LLRG is explored in Sect. 5. The results are discussed and summarised in Sect. 6.

We adopted $H_{0}=75 \mathrm{~km} \mathrm{~s}^{-1} \mathrm{Mpc}^{-1}$ and $q_{0}=0.5$.

\section{Chandra data analysis}

We selected the same sample considered by Chiaberge et al. (1999) formed by the 33 radio-sources from the 3CR catalogue with FR I morphology ${ }^{1}$.

We searched for Chandra observations available in the public archive up to January 2005 and found data for 18 objects (see Table 1). All observations we considered were made using the Advanced CCD Imaging Spectrometer (ACIS-I and ACIS-S) without any transmission grating in place. When more than one observation was available, we usually chose the one with the longer exposure times; only for 3C 338 were two observations of approximately equal length present and fitted simultaneously. For the brightest objects we instead chose the observation with the lowest time frame to reduce the pile-up effect.

We reduced all the data using the Chandra data analysis CIAO v3.0.2, with the CALDB version 2.25. Therefore, to correct for the QE degradation, we created a new ARF (effective area files) using the new file "acisD1999-08-13contamN0003.fits" released with the CALDB version 2.26 (as recommended by the "ACIS Modeling and Analysis Team"). We reprocessed all the data from level 1 to level 2, using the CIAO tool acis_process_event with all parameters restored to the default values, i.e. applying the pixels and PHA randomization. The new event file was

1 The only addition is 3C 189 , which is an FR I part of the 3C sample (Edge et al. 1959) but not of its revised version 3CR (Bennett 1962). 
Table 2. Results of the fit: (1) source name, (2) Galactic hydrogen column density $\left[10^{22} \mathrm{~cm}^{-2}\right]$, (3) local hydrogen column density $\left[10^{22} \mathrm{~cm}^{-2}\right]$, (4) power-law photon index $\Gamma$, (5) temperature of the thermal component $[\mathrm{keV}]$, (6) observed flux of the thermal component corrected for Galactic absorption [erg cm $\mathrm{cm}^{-2} \mathrm{~s}^{-1}$ ], (7) non-thermal nuclear flux (corrected for absorption) at $1 \mathrm{keV}$ [erg $\mathrm{cm}^{-2} \mathrm{~s}^{-1}$ ], (8) $\chi^{2} / \mathrm{degrees}$ of freedom. When the number of d.o.f. was smaller than 10 , no fit was performed. The $*$ mark sources affected by pile-up and corrected with the Davies's algorithm.

\begin{tabular}{|c|c|c|c|c|c|c|c|}
\hline Name & $N_{\mathrm{H}, \mathrm{gal}}$ & $N_{\mathrm{H}}^{z}$ & $\Gamma$ & $K T$ & $F_{\mathrm{x}, \text { ther }}(0.5-5 \mathrm{keV})$ & $F_{\mathrm{x}, \text { nuc }}(1 \mathrm{keV})$ & $\chi^{2} /$ d.o.f. \\
\hline $3 \mathrm{C} 028$ & 0.05 & - & - & - & - & $<1.5 \mathrm{E}-14$ & $-/ 0$ \\
\hline 3C 031 & 0.05 & $<0.3$ & $1.4_{-0.4}^{+0.3}$ & $0.6_{-0.1}^{+0.1}$ & $3.3_{-0.7}^{+0.8} \mathrm{E}-14$ & $1.9_{-0.5}^{+1.0} \mathrm{E}-14$ & $29.4 / 24$ \\
\hline $3 \mathrm{C} 066 \mathrm{~B}$ & 0.09 & $<0.7$ & $2.4_{-0.4}^{+0.7}$ & $0.3_{-0.1}^{+0.1}$ & $6.2_{-2.7}^{+1.4} \mathrm{E}-14$ & $1.2_{-0.4}^{+0.8} \mathrm{E}-13$ & $30.3 / 37$ \\
\hline $3 \mathrm{C} 075$ & 0.09 & - & - & - & - & $<3.6 \mathrm{E}-14$ & $-/ 2$ \\
\hline $3 \mathrm{C} 078^{*}$ & 0.10 & $<0.4$ & $2.2_{-0.3}^{+0.4}$ & $0.2_{-0.1}^{+0.1}$ & $1.6_{-1.5}^{+0.8} \mathrm{E}-13$ & $7.4_{-4.1}^{+4.6} \mathrm{E}-13$ & $39.4 / 27$ \\
\hline 3C 083.1 & 0.15 & $2.5_{-1.4}^{+0.8}$ & $2.2_{-0.5}^{+0.9}$ & $0.5_{-0.1}^{+0.1}$ & $2.7_{-0.3}^{+0.7} \mathrm{E}-14$ & $6.3_{-4.1}^{15.2} \mathrm{E}-14$ & $7.19 / 19$ \\
\hline $3 \mathrm{C} 084^{*}$ & 0.15 & $<0.1$ & $1.6_{-0.1}^{+0.1}$ & $0.3_{-0.1}^{+0.1}$ & $7.6_{-1.6}^{+3.5} \mathrm{E}-13$ & $2.8_{-0.1}^{+0.5} \mathrm{E}-12$ & $150.1 / 161$ \\
\hline 3C 189 & 0.05 & $<0.2$ & $2.1_{-0.4}^{+0.6}$ & $0.3_{-0.1}^{+0.2}$ & $4.5_{-3.4}^{+5.4} \mathrm{E}-14$ & $1.3_{-0.2}^{+0.5} \mathrm{E}-13$ & $22.7 / 16$ \\
\hline $3 \mathrm{C} 270$ & 0.01 & $5.7_{-3.4}^{+3.2}$ & $0.8_{-0.4}^{+0.7}$ & $0.5_{-0.1}^{+0.1}$ & $6.6_{-0.6}^{+0.7} \mathrm{E}-14$ & $8.4_{-5.4}^{13.3} \mathrm{E}-14$ & $54.15 / 50$ \\
\hline 3C 272.1 & 0.02 & $0.2_{-0.1}^{+0.3}$ & $2.1_{-0.3}^{+0.2}$ & $0.5_{-0.2}^{+0.3}$ & $1.0_{-0.7}^{+1.3} \mathrm{E}-14$ & $5.1_{-1.8}^{+3.7} \mathrm{E}-14$ & $17.9 / 23$ \\
\hline $3 \mathrm{C} 274^{*}$ & 0.02 & $<0.03$ & $2.3_{-0.1}^{+0.1}$ & $0.7_{-0.1}^{+0.1}$ & $1.2_{-0.4}^{+0.4} \mathrm{E}-13$ & $7.2_{-0.5}^{+0.9} \mathrm{E}-13$ & $118.6 / 98$ \\
\hline $3 \mathrm{C} 296$ & 0.02 & $1.0_{-0.6}^{0.6}$ & $1.6_{-0.4}^{+0.5}$ & $0.5_{-0.1}^{+0.1}$ & $3.2_{-0.4}^{+0.3}$ E-14 & $6.5_{-2.0}^{+3.4} \mathrm{E}-14$ & $27.81 / 33$ \\
\hline $3 \mathrm{C} 317$ & 0.03 & $<0.1$ & $2.0_{-0.2}^{+0.2}$ & $0.2_{-0.1}^{+0.2}$ & $1.1_{-0.9}^{+0.8} \mathrm{E}-14$ & $6.3_{-0.7}^{+0.9} \mathrm{E}-14$ & $33.6 / 38$ \\
\hline $3 C 338$ & 0.01 & - & - & - & - & $<3.3 \mathrm{E}-14$ & $-/ 8$ \\
\hline $3 \mathrm{C} 346$ & 0.05 & $<0.1$ & $1.7_{-0.1}^{+0.1}$ & $0.6_{-0.3}^{+0.6}$ & $1.5_{-1.1}^{+1.1} \mathrm{E}-14$ & $1.5_{-0.1}^{+0.1} \mathrm{E}-13$ & $92.1 / 89$ \\
\hline 3C 348 & 0.06 & - & - & - & - & $<3.1 \mathrm{E}-14$ & $-/ 0$ \\
\hline $3 \mathrm{C} 438$ & 0.21 & - & - & - & - & $<1.5 \mathrm{E}-14$ & $-/ 0$ \\
\hline $3 \mathrm{C} 449$ & 0.11 & - & - & - & - & $<2.1 \mathrm{E}-14$ & $-/ 0$ \\
\hline
\end{tabular}

filtered by selecting a standard set of ASCA grades (02346) and rejecting time intervals with high background levels.

Our aim was the study of the X-ray emission associated to the AGN. For this reason we chose as extracting region a circle of 1 .'2 radius centred on the emission peak (for the fainter nuclei we checked their position using HST or VLA observation) and as background region a source-centred annulus with radii between 1 '.2-2'.5. When X-ray emission from an extended jet is present, we excluded the corresponding angular portion from the background region. With this strategy we aimed to reduce the diffuse thermal X-ray component as much as possible, so as to maximize the nuclear contribution.

A possible drawback of this approach, i.e. a small extraction region, is related to the variations in the Point Spread Function with energy. These might cause the inclusion of a varying fraction of the nuclear emission in our aperture in the different bands. However, we tested that the PSF effects were negligible. In particular the fraction of nuclear counts falling in the background annulus decreases only from $\sim 8 \%$ in the $0.5-2 \mathrm{keV}$ band to $\sim 2 \%$ in the $2-5 \mathrm{keV}$ band. This has a marginal impact on the derived luminosities and spectral indices.

The spectra were modeled using XSPEC version 11.3.0. We used only events with energy $>0.5 \mathrm{keV}$, where the calibration is best known and the background contribution is negligible. We rebinned the spectrum to a minimum of 25 counts per bin in order to ensure the applicability of the $\chi^{2}$ statistics. We then modeled all the spectra with a superposition of a thermal and a power-law (non-thermal) component. For the thermal component the metal abundances were fixed to 0.5 (Fabbiano 1989) and the absorption was set to the galactic value; for the power-law component we allowed for the presence of local absorption. The relative complexity of the model prevents us from obtaining a robust estimate of all free parameters for the sources with a low count rate. More quantitatively, we experimented that the fit is sufficiently constrained in the twelve objects for which the spectrum can be regrouped in at least 15 channels, so as to leave us with $\gtrsim 10$ degree of freedom, corresponding to approximately $\sim 400$ total net counts. In the six sources below this threshold, we set upper limits for the non-thermal fluxes and fit their spectra using only a power-law model (with column density fixed at the galactic level). We initially fixed the power law index to 2 but, to be conservative, we then looked for the value of Gamma in the range 1.5-2.5 corresponding to the largest flux value.

This is a quite conservative approach but our choice was supported by the fact that in these objects we cannot accurately disentangle the contribution of the thermal and non-thermal components; a less cautious analysis is prone to highly uncertain estimates, in particular of the AGN X-ray flux. For the 3 brightest sources (3C 78, 3C 84, and 3C 274) we included the correction for the effects of the pile-up by applying the algorithm proposed by Davis (2001). The results of the fit are given in Table 2.

For the 12 objects in which we performed a two-component fit, we used with an F-test ${ }^{2}$ to verify that the presence of both

\footnotetext{
${ }^{2}$ Protassov et al. (2002) suggested that it might be inappropriate to use the F-test to test an added spectral component. However, because at the moment no other simple, alternative method is known, we decided to apply the F-test in any case, above all considering that the presence of an extended thermal emission and a point-like nuclear source are also directly proved by the image.
} 
Table 3. Summary of radio, optical and X-ray data of the sample: (1) source name, (2) redshift of the galaxy, (3) nuclear radio flux at $5 \mathrm{GHz}$ [mJy], (4) nuclear radio luminosity at $5 \mathrm{GHz}$ [erg s${ }^{-1}$ ], (5) extended radio luminosity at $178 \mathrm{MHz}$ [erg s${ }^{-1}$ ], (6) optical nuclear luminosity at $5500 \AA$ [erg s$\left.{ }^{-1}\right]$, (7) unabsorbed X-ray nuclear flux $(0.5-5 \mathrm{keV})$ [ $\left.\mathrm{erg} \mathrm{cm}^{-2} \mathrm{~s}^{-1}\right]$, (8) X-ray nuclear luminosity $(0.5-5 \mathrm{keV})$ corrected for absorption [erg s ${ }^{-1}$ ]. Information about redshift, radio core flux, extended radio emission, optical luminosities are from Chiaberge et al. (1999). We adopt an optical spectral index $\alpha_{\mathrm{o}}=1$.

\begin{tabular}{lrrrrrrr}
\hline \hline Name & Redshift & $F_{\mathrm{c}}(5 \mathrm{GHz})$ & $v L_{\mathrm{c}}(5 \mathrm{GHz})$ & $v L_{\mathrm{t}}(178 \mathrm{MHz})$ & $v L_{\mathrm{o}}$ & $F_{\mathrm{x}}(0.5-5 \mathrm{keV})$ & $L_{\mathrm{x}}(0.5-5 \mathrm{keV})$ \\
\hline 3C 028 & 0.1952 & $<0.2$ & $<7.3 \mathrm{E} 38$ & $2.1 \mathrm{E} 42$ & $<1.3 \mathrm{E} 41$ & $<3.4 \mathrm{E}-14$ & $<2.5 \mathrm{E} 42$ \\
3C 031 & 0.0169 & 92.0 & $2.5 \mathrm{E} 39$ & $1.6 \mathrm{E} 40$ & $4.5 \mathrm{E} 40$ & $6.2_{-1.7}^{+3.1} \mathrm{E}-14$ & $3.4_{-0.1}^{+0.2} \mathrm{E} 40$ \\
3C 066B & 0.0215 & 182.0 & $8.0 \mathrm{E} 39$ & $3.8 \mathrm{E} 40$ & $2.4 \mathrm{E} 41$ & $2.4_{-0.8}^{+1.6} \mathrm{E}-13$ & $2.1_{-0.1}^{+0.1} \mathrm{E} 41$ \\
3C 075 & 0.0232 & 39.0 & $2.0 \mathrm{E} 39$ & $1.9 \mathrm{E} 40$ & - & $<8.3 \mathrm{E}-14$ & $<8.5 \mathrm{E} 40$ \\
3C 078 & 0.0288 & 964.0 & $7.6 \mathrm{E} 40$ & $5.5 \mathrm{E} 40$ & $2.1 \mathrm{E} 42$ & $8.8_{-3.6}^{+1.5} \mathrm{E}-13$ & $1.4_{-0.1}^{+0.1} \mathrm{E} 42$ \\
3C 083.1 & 0.0251 & 21.0 & $1.3 \mathrm{E} 39$ & $5.7 \mathrm{E} 40$ & $9.3 \mathrm{E} 39$ & $1.3_{-0.9}^{+3.1} \mathrm{E}-13$ & $1.6_{-0.1}^{0.3} \mathrm{E} 41$ \\
3C 084 & 0.0176 & 42370.0 & $1.2 \mathrm{E} 42$ & $4.2 \mathrm{e} 40$ & $4.9 \mathrm{E} 42$ & $6.1_{-0.1}^{+0.8} \mathrm{E}-12$ & $3.6_{-0.1}^{+0.1} \mathrm{E} 42$ \\
3C 189 & 0.043 & 195 & $3.4 \mathrm{E} 40$ & $7.1 \mathrm{E} 40$ & $6.2 \mathrm{E} 41$ & $2.8_{-0.6}^{+1.1} \mathrm{E}-13$ & $9.9_{-0.1}^{+0.1} \mathrm{E} 41$ \\
3C 270 & 0.0074 & 308.0 & $1.6 \mathrm{E} 39$ & $1.0 \mathrm{E} 40$ & $2.9 \mathrm{E} 39$ & $4.4_{-2.9}^{+6.9} \mathrm{E}-13$ & $4.6_{-0.3}^{+0.8} \mathrm{E} 40$ \\
3C 272.1 & 0.0037 & 180.0 & $2.3 \mathrm{E} 38$ & $9.0 \mathrm{E} 38$ & $8.5 \mathrm{E} 39$ & $1.5_{-0.4}^{+0.8} \mathrm{E}-13$ & $3.9_{-0.1}^{+0.3} \mathrm{E} 39$ \\
3C 274 & 0.0037 & 4000.0 & $5.2 \mathrm{E} 39$ & $4.9 \mathrm{E} 40$ & $5.6 \mathrm{E} 40$ & $1.3_{-0.1}^{+0.2} \mathrm{E}-12$ & $3.4_{-0.1}^{+0.1} \mathrm{E} 40$ \\
3C 296 & 0.0237 & 77.0 & $4.1 \mathrm{E} 39$ & $2.5 \mathrm{E} 40$ & $2.0 \mathrm{E} 40$ & $1.8_{-0.5}^{+1.0} \mathrm{E}-13$ & $1.9_{-0.1}^{+0.1} \mathrm{E} 41$ \\
3C 317 & 0.0342 & 391.0 & $4.4 \mathrm{E} 40$ & $1.9 \mathrm{E} 41$ & $1.2 \mathrm{E} 41$ & $1.5_{-0.2}^{+0.2} \mathrm{E}-13$ & $3.3_{-0.1}^{+0.1} \mathrm{E} 41$ \\
3C 338 & 0.0303 & 105.0 & $9.2 \mathrm{E} 39$ & $1.4 \mathrm{E} 41$ & $9.7 \mathrm{E} 40$ & $<7.6 \mathrm{E}-14$ & $<1.3 \mathrm{E} 41$ \\
3C 346 & 0.1620 & 220.0 & $5.5 \mathrm{E} 41$ & $9.7 \mathrm{E} 41$ & $6.4 \mathrm{E} 42$ & $4.0_{-0.3}^{+0.2} \mathrm{E}-13$ & $2.0_{-0.1}^{+0.1} \mathrm{E} 43$ \\
3C 348 & 0.1540 & 10. & $2.3 \mathrm{E} 40$ & $2.8 \mathrm{E} 43$ & $2.0 \mathrm{E} 41$ & $<7.3 \mathrm{E}-14$ & $<3.3 \mathrm{E} 42$ \\
3C 438 & 0.2900 & 17.0 & $1.4 \mathrm{E} 41$ & $1.3 \mathrm{E} 43$ & $<3.5 \mathrm{E} 41$ & $<3.4 \mathrm{E}-14$ & $<5.4 \mathrm{E} 42$ \\
3C 449 & 0.0181 & 37.0 & $1.1 \mathrm{E} 39$ & $1.3 \mathrm{E} 40$ & $6.2 \mathrm{E} 40$ & $<4.8 \mathrm{E}-14$ & $<3.0 \mathrm{E} 40$ \\
\hline
\end{tabular}

a thermal and non-thermal component always corresponded to an improvement of the fit at a level $>95 \%$.

Errors and upper limits are quoted at $90 \%$ confidence for one parameter of interest $\left(\Delta \chi^{2}=2.71\right)$, with the exception of $N_{\mathrm{H}}^{z}$ and $\Gamma$, which are well known to be strongly coupled and we used a $90 \%$ confidence level for two parameters of interest $\left(\Delta \chi^{2}=4.61\right)$.

Donato et al. (2004) considered X-ray observations of a sample of low luminosity radio-galaxies, largely overlapping with the objects discussed here. With the exception of 3C 338, 3C 348, and 3C 449, which we more conservatively defined as upper limits, there is complete agreement between the two lists of detected X-ray nuclei. Also the temperature of the thermal component, as well as the power-law spectral index, is in very good accord when they are measured from Chandra data. Considering the values of $N_{\mathrm{H}}^{z}$, and since we treated more conservatively the errors on this quantity, we obtained upper limits in a few objects that Donato et al. considered as detection of local absorption.

\section{Results}

Before we proceed with a discussion of our results, it is important to note that, since archival data are available for a fraction of about half of the original sample of $3 \mathrm{C} / \mathrm{FR} \mathrm{I}$, it is possible that we are not considering an unbiased sub-sample. We therefore compared various parameters (e.g. redshift, core and total radio-power) for the $3 \mathrm{C} / \mathrm{FR}$ I sample as a whole and for our sub-sample. Applying a Kolmogorov-Smirnov test, we found that their distributions are consistent with being drawn from the same population with the probability always higher than $60 \%$. The fraction of optically nucleated galaxies in the Chandra subsample is slightly larger, $83 \%$ compared to a $70 \%$ for the whole sample (Chiaberge et al. 1999). Overall, it appears that the galaxies studied here are representat the entire 3C/FR I sample well. On the other hand, several galaxies with somewhat peculiar radio-morphology are excluded, (e.g. the fat double $3 \mathrm{C} 314.1$, the compact radio-source associated to $3 \mathrm{C} 293$, the $\mathrm{X}$-shaped 3C 305, or the complex radio-source in 3C 433) possibly leading to a higher fraction of objects with classical corejets FR I morphology.

\subsection{Local absorption in X-ray nuclei}

We obtained a statistically significant estimate for the local column density in four objects (namely 3C 83.1, 3C 270, 3C 272.1, and 3C 296) with values $N_{\mathrm{H}}^{z} \sim 0.2-6 \times 10^{22} \mathrm{~cm}^{-2}$. In the other cases (8/12) we find an upper limit to $N_{\mathrm{H}}^{z}$ (Table 2) ranging from $0.03 \times 10^{22} \mathrm{~cm}^{-2}$ to $0.7 \times 10^{22} \mathrm{~cm}^{-2}$. In Fig. 1 we present a histogram of the values of the intrinsic absorption: the white rectangles represent upper limits, while the black ones are the four detections.

\subsection{Relations between radio, optical, and X-ray emission}

In Fig. 2 (top left panel) we compare the nuclear fluxes in the radio and X-ray bands. A strong correlation is present: the generalized (including the presence of upper limits) Spearman rank 


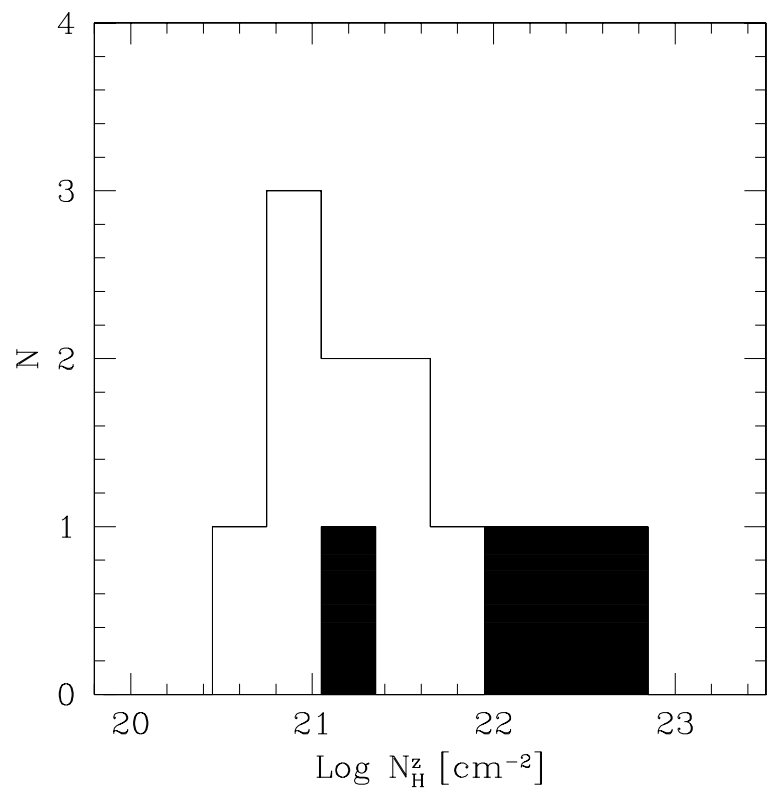

Fig. 1. Histogram of intrinsic absorption in $\log N_{\mathrm{H}}^{z}$. The black rectangles represent detections, while the white ones represent upper limits (at $90 \%$ confidence level).

correlation coefficient $\rho=0.88$ corresponding to a probability that the correlation is not present of $P=0.001$.

The best linear fits were derived as the bisectrix of the fits using the two quantities as independent variables following the suggestion by Isobe et al. (1990) that this is preferable for problems needing symmetrical treatment of the variables. The presence of upper limits in the independent variable suggests taking advantage of the methods of survival analysis proposed by e.g. Schmitt (1985). However, the drawbacks discussed by Sadler et al. (1989) and, in our specific case, also the non-random distribution of upper limits, disfavour this approach. We therefore preferred to exclude upper limits from the linear regression analysis. Nonetheless we note that, a posteriori, 1) the objects with undetected nuclear component in the X-ray are consistent with the correlation defined only by the detections; 2) the application of the Schmidt methods provides correlation slopes that agree, within the errors, with our estimates.

A similarly tight correlation between radio and X-ray nuclear emission is also present considering luminosities, with $\rho=0.72$ corresponding to a probability $P=0.003$ (see Fig. 2, top right panel); it extends over 4 orders of magnitude, with a slope of $m=0.91 \pm 0.12$ and a dispersion of $0.39 \mathrm{dex}$ (see Table 4 for a summary of the statistical analysis). We tested the possible influence of redshift in driving this correlation (both quantities depend on $z^{2}$ ) estimating the Spearman partial rank coefficient ${ }^{3}$. The effect is negligible as the value of $\rho$ is essentially unchanged.

We find a similarly strong connection between the X-ray versus optical nuclear luminosity (Fig. 2, left bottom panel).

\footnotetext{
3 The Spearman Partial Rank correlation coefficient estimates the linear correlation coefficient between two variables taking the presence of a third into account. If $\mathrm{A}$ and $\mathrm{B}$ are both related to the variable $z$, the Spearman correlation coefficient is $\rho_{\mathrm{AB}, z}=\frac{\rho_{\mathrm{AB}}-\rho_{\mathrm{A} z} \rho_{\mathrm{B} z}}{\left[\left(1-\rho_{\mathrm{A} z}^{2}\right)\left(1-\rho_{\mathrm{B} z}^{2}\right)\right]^{1 / 2}}$.
}

Again, all X-ray upper limits are consistent with the $L_{\mathrm{O}}$ vs. $L_{\mathrm{X}}$ correlation defined by the detected objects. Conversely, note that all three sources where we measured the largest X-ray absorbing column densities (namely 3C 83.1, 3C 270, and 3C 296) lie significantly above the correlation between $L_{O}$ and $L_{\mathrm{X}}$. This is not surprising since, while the estimated X-ray fluxes are de-absorbed, the optical measurements are not corrected for the presence of local absorption. Indeed, the location of these X-ray absorbed sources in the $L_{O}-L_{X}$ is suggestive of significant absorption also in the optical band. We therefore preferred to re-estimate the correlation omitting these three objects, shown in Fig. 2 with a solid line. We obtained a smaller dispersion of 0.23 dex and a steeper corrected slope, $m=1.16 \pm 0.09$.

In the radio-optical plane $L_{\mathrm{r}}-L_{\mathrm{O}}$ we also found a strong correlation as already found by Chiaberge et al. (1999), see Fig. 2, bottom right panel. Again, the 3 X-ray absorbed sources are located below the best fit, supporting the interpretation of an optical deficit derived from their location in the $L_{\mathrm{O}}-L_{\mathrm{X}}$ plane. Similarly, we re-estimated the correlation excluding the potentially absorbed objects in the optical. The slope of the corrected correlation is $m=0.82 \pm 0.11$ (very similar to the slope of the correlation found by Chiaberge et al. (1999), $m=0.88 \pm 0.12$, using the whole 3C/FR I sample) with a dispersion of 0.32 dex.

\section{The X-ray absorbing medium in FR I nuclei}

In 4 galaxies we obtained a detection of local X-ray absorption. In all cases our estimates of the column density agrees closely with previously published measurements ${ }^{4}$. The inspection of their HST images (see Fig. 3) is particularly instructive as they unveil a connection between optical and X-ray absorption. The three sources for which we found the largest X-ray absorption show circumnuclear dusty disk structures, on scales between $300 \mathrm{pc}-1 \mathrm{kpc}$. All disks are seen at large inclinations, the less inclined being associated to 3C 270 whose axis forms an angle $\theta \geq 64^{\circ}$ with the line of sight (Jaffe et al. 1996). The fourth X-ray absorbed galaxy is 3C 272.1: its HST image show the presence of a low optical depth $\left(A_{\mathrm{V}} \sim 0.54\right.$, Bower et al. 1997) filamentary dust lane, directly projected onto the nucleus.

Dust is by no means exclusively found in these 4 sources. The HST images of the remaining radio-galaxies also show the presence of dust, even in the form of disks. For example, 3C 66B and 3C 78 have face-on disks (Sparks et al. 2000), while disks at higher inclinations (but still substantially less inclined than in the 3 cases discussed above) are found in 3C 31 and $3 \mathrm{C}$ 449. Off-nuclear dust, usually distributed in filaments, is also seen in $3 \mathrm{C} 84,3 \mathrm{C} 274,3 \mathrm{C} 317$, and 3C 338. In most of these sources, the quality of the $\mathrm{X}$-ray spectra is sufficient for setting stringent limits on the column density, $N_{\mathrm{H}}<10^{22} \mathrm{~cm}^{-2}$.

Therefore it appears that X-ray absorption is associated with the presence of dust, but only when i) the dust intercepts

\footnotetext{
4 See Chiaberge et al. (2002) and Donato et al. (2004) for 3C 270; Harris et al. (2002) and Donato et al. (2004) for 3C 272.1; Hardcastle et al. (2005) for 3C 296.
} 

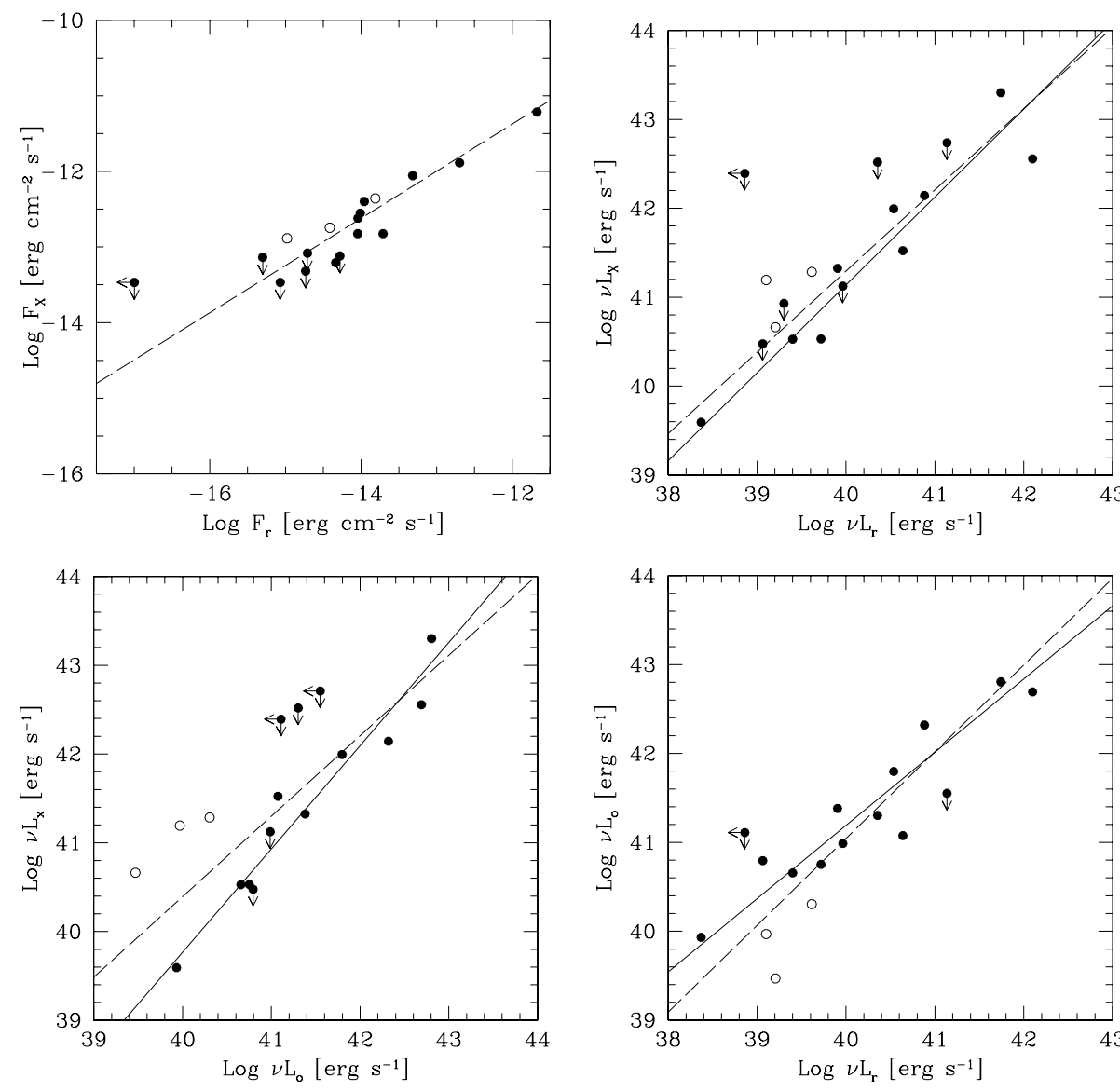

Fig. 2. Comparison of (top left) radio $(5 \mathrm{GHz})$ and $\mathrm{X}$-ray (0.5-5 keV) nuclear fluxes, (top right) radio and $\mathrm{X}$-ray luminosities, (bottom left) optical $(5500 \AA)$ and X-ray luminosities, (bottom right) optical and radio luminosities. The empty circles represent the objects for which we have detected an intrinsic absorption $>10^{22} \mathrm{~cm}^{-2}$. The solid line reproduces the best linear fit after having excluded the 3 X-ray absorbed nuclei, while 3 the dashed line is the fit on the whole sample.

Table 4. Correlations summary.

\begin{tabular}{llcccc}
\hline \hline Var. A & Var. B & $\rho_{\mathrm{AB}}(\mathrm{P})$ & $\rho_{\mathrm{AB}, z}$ & Slope & rms \\
\hline$F_{\mathrm{X}}$ & $F_{\mathrm{r}}$ & $0.88(0.001)$ & - & $0.62 \pm 0.14$ & 0.27 \\
$L_{\mathrm{X}}$ & $L_{\mathrm{r}}$ & $0.72(0.003)$ & 0.67 & $0.91 \pm 0.12$ & 0.39 \\
$L_{\mathrm{x}}$ & $L_{\mathrm{r}}$ corr & $0.79(0.003)$ & 0.77 & $0.99 \pm 0.11$ & 0.33 \\
$L_{\mathrm{x}}$ & $L_{\mathrm{o}}$ & $0.71(0.004)$ & 0.67 & $0.91 \pm 0.16$ & 0.52 \\
$L_{\mathrm{x}}$ & $L_{\mathrm{o}}$ corr & $0.86(0.002)$ & 0.85 & $1.16 \pm 0.09$ & 0.23 \\
$L_{\mathrm{o}}$ & $L_{\mathrm{r}}$ & $0.79(0.002)$ & 0.75 & $0.98 \pm 0.11$ & 0.39 \\
$L_{\mathrm{o}}$ & $L_{\mathrm{r}}$ corr & $0.79(0.005)$ & 0.76 & $0.82 \pm 0.11$ & 0.32 \\
\hline
\end{tabular}

$\rho_{\mathrm{AB}}$ is the generalized Spearman's correlation coefficient (computed including upper limits) and in parenthesis the probability that there is no correlation between the variables. $\rho_{\mathrm{AB}, z}$ is the partial rank coefficient after excluding on the common dependence of luminosities $\mathrm{A}$ and $\mathrm{B}$ on redshift. The $3 \mathrm{X}$-ray absorbed nuclei are excluded in the analysis marked with "corr".

our line of sight to the nucleus or ii) it takes the form of a highly inclined disk. While the first case is rather obvious, the second situation $^{5}$ indicates that the gas responsible for the X-ray absorption shares the flattened distribution of the dust, inducing a dependence of $N_{\mathrm{H}}$ on the viewing angle. Note that evidence of a dependence of absorption on orientation in FR I nuclei was

5 This behaviour is also shared by the radio-galaxy B2 $0055+30$, with a large column density $N_{\mathrm{H}} \sim 7 \times 10^{22} \mathrm{~cm}^{-2}$ (Donato et al. 2004) associated to a highly inclined disk in the HST image (Capetti et al. 2000a). already found by Chiaberge et al. (2002) from the comparison of the optical and UV images of radio-galaxies.

However, the column densities toward FR I nuclei are much smaller than those of the optical and X-ray absorbers predicted (and required) by the AGN unified models, i.e. the circumnuclear obscuring tori; as a comparison, in the obscured Seyfert 2 galaxies $75 \%$ have $N_{\mathrm{H}}>10^{23} \mathrm{~cm}^{-2}$ and about half of them are Compton thick $\left(N_{\mathrm{H}}>10^{24} \mathrm{~cm}^{-2}\right.$, Risaliti et al. 1999). Unfortunately, we cannot constrain the location of the X-ray absorbing gas, other than it must be coplanar with the largescale dust. It might be also cospatial with the dust and thus being distributed on a kpc scale, rather than on the characteristic pc scale of tori in Seyfert galaxies.

Let's now focus on the three sources for which we found the largest X-ray absorption. In Sect. 2 we noted that their location with respect to the $L_{\mathrm{O}}$ vs. $L_{\mathrm{X}}$, as well as $L_{\mathrm{O}}$ vs. $L_{\mathrm{r}}$ correlations can be interpreted as due to absorption in the optical band ${ }^{6}$. The optical absorption can be estimated quantitatively from their offsets from the best fit relations. We find $A_{R} \sim 2-4$ from the $L_{\mathrm{O}}$ vs. $L_{\mathrm{X}}$ plane and slightly lower values, $A_{R} \sim 1.5-3$, from the $L_{\mathrm{O}}$ vs. $L_{\mathrm{R}}$ plane. The ratios between optical and X-ray absorption are in the range $A_{V} / N_{\mathrm{H}} \sim(0.7-3.3) \times 10^{-22} \mathrm{mag} \mathrm{cm}{ }^{2}$, having transformed $A_{V}=1.34 A_{R}$ using the Cardelli et al. (1989) extinction law. These values are slightly smaller than the standard galactic ratio, $A_{V} / N_{\mathrm{H}} \sim 5 \times 10^{-22} \mathrm{mag} \mathrm{cm}^{2}$

\footnotetext{
6 This interpretation is supported by the extremely steep UV/optical nuclear spectrum of 3C 270 (Capetti et al. 2000b), which must be ascribed to absorption.
} 

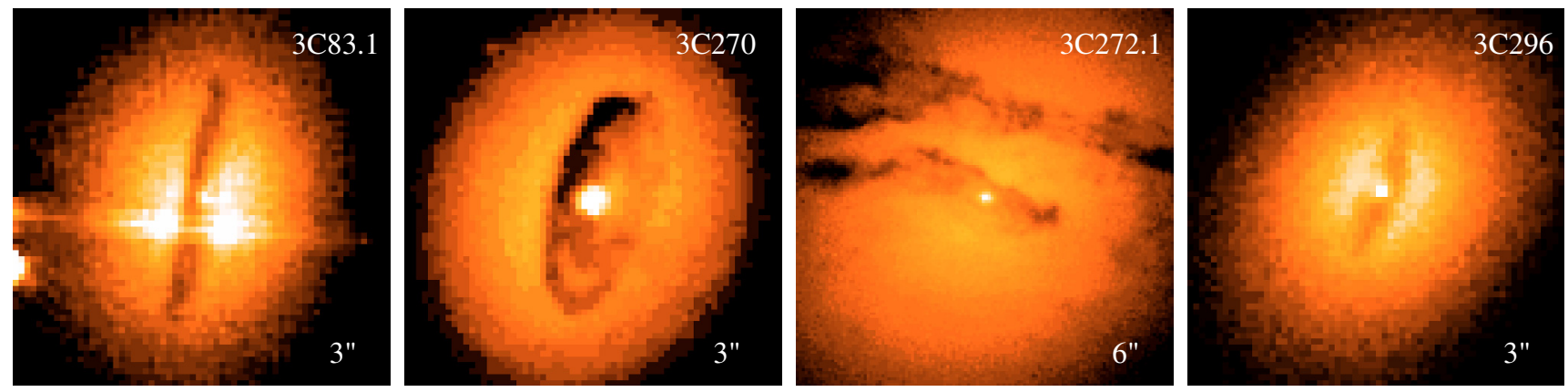

Fig. 3. HST images of the 4 radio-galaxies in which we detected a local X-ray absorption, taken from Chiaberge et al. (1999).

(Bohlin et al. 1978). Maiolino et al. (2001) also found evidence for a low $A_{V} / N_{\mathrm{H}}$ ratio in $\mathrm{AGN}$, but with values extending down to values as low as $A_{V} / N_{\mathrm{H}} \sim 3 \times 10^{-24} \mathrm{mag} \mathrm{cm}^{2}$. They interpreted this finding as evidence for dust in the circumnuclear region of AGNs that is different from Galactic dust, possibly due to the dominance of larger grains. Clearly, our results indicate a much less extreme departure for the $A_{V} / N_{\mathrm{H}}$ value and consequently from the galactic dust properties.

The fraction of X-ray absorbed sources also sets a limit to the gas covering factor. Three out of 12 objects (25\%) are associated to a column density larger than $10^{22} \mathrm{~cm}^{-2}$. As a reference value, the fraction of obscured objects in the CfA sample of Seyfert galaxies is $53 \%$, considering only type 2 (Huchra \& Burg 1992), but it might be as high as $78 \%$ if the intermediate types 1.8 and 1.9 are also included (Osterbrock \& Martel 1993). Given the small (and incomplete) sample statistics and the different (and somewhat arbitrary) definition of "absorbed" FR I or Seyfert, a direct comparison is probably not appropriate. Although X-ray absorption in FR I is associated to low column densities, it is not certainly a rare phenomenon.

\section{A jet-origin for the X-ray cores?}

The central issue for our understanding of the properties of LLRG is the origin of their nuclear emission. Analysis of the results presented in Sect. 3.2 provides several pieces of evidence, as presented in the following three subsections, supporting the interpretation that the radio, optical, and X-ray cores have a common origin, most likely being produced by the base of a relativistic jet, as already suggested from the analysis of ROSAT and HST data by Worrall \& Birkinshaw (1994) and Chiaberge et al. (1999).

\subsection{Multiwavelength nuclear correlations}

The first indication comes from the presence of the strong correlations between the nuclear luminosities in the three available bands.

A connection between the radio, optical, and X-ray radiation is also observed in other classes of AGNs including Seyfert galaxies, radio-galaxies, and QSO (see e.g. Falcke et al. 1995). However, the dispersion between the emission in the different bands is substantially larger than observed in our sample. For example, at a given radio luminosity, the $\mathrm{X}$-ray emission spans 4-5 orders of magnitude; even projecting this correlation in a plane when also considering the different black hole masses, the rms is still as large as $0.88 \mathrm{dex}$ (Merloni et al. 2003). These results show, on the one hand, the close link between accretion and ejection in AGN but, on the other, indicate the relatively large allowed range for the parameters coupling the two processes and their radiative manifestations, e.g. the strength of the magnetic field and the structure of the accretion disk.

Conversely, the rms of the data points for our FR I sample around the best fit are typically a mere factor of 2 , despite the large range of luminosities involved. Furthermore, concerning the radio- $X$ correlation, it must also be noted that part of the dispersion certainly arises from the (often substantial) errors associated to (at least) the X-ray luminosities, see Table 2. If one considers that other effects also contribute to the dispersion (e.g. variability in non simultaneous observations), the rms value must be considered as a strict upper limit to the intrinsic dispersion.

Thus our results radically differ from a quantitative point of view from the general trend found in other AGNs and point to a much closer connection, such as a common origin for the radio, optical, and $\mathrm{X}$-ray emission.

\subsection{Broad band spectral indices}

The issue of the origin of the X-ray emission in FR I can also be addressed concentrating on the Spectral Energy Distributions (SED) of FR I compared to those of other classes of AGNs. As SED behaviour can be parameterized using the broad-band spectral indices, we combined the available data in the different energy bands (scaled to the standard reference values, i.e. the radio at $5 \mathrm{GHz}$, the optical at $5500 \AA$ and $\mathrm{X}$-ray luminosity at $1 \mathrm{keV}$ ) and we calculate the broad-band spectral indices of our objects. They are presented in Table 5 and reproduced graphically in the ( $\alpha_{\text {ox }}$ vs. $\left.\alpha_{\text {ro }}\right)$ plane (see Fig. 4$)$. The distribution of spectral indices completly agrees with the results obtained by Hardcastle \& Worrall (2000), who used ROSAT data for the X-ray measurements.

All FR I sources are confined in the upper left region of this plane and show a remarkable homogeneity in their spectral shapes with $\alpha_{\mathrm{ox}}=1.13 \pm 0.11, \alpha_{\mathrm{ro}}=0.77 \pm 0.08$, and $\alpha_{\mathrm{rx}}=$ $0.90 \pm 0.05$. The quoted uncertainties represent the measured dispersions of each index, not considering the X-ray absorbed sources. These objects are slightly offset from the remainder of 
Table 5. Broad-band spectral indices.

\begin{tabular}{lccc}
\hline \hline Name & $\alpha_{\text {ox }}$ & $\alpha_{\text {ro }}$ & $\alpha_{\text {rx }}$ \\
\hline 3C 028 & - & - & - \\
3C 031 & $1.24_{-0.33}^{+0.24}$ & $0.75 \pm 0.12$ & $0.92_{-0.07}^{+0.01}$ \\
3C 066B & $1.13_{-0.29}^{+0.58}$ & $0.71 \pm 0.01$ & $0.85_{-0.10}^{+0.20}$ \\
3C 075 & - & - & $>0.83$ \\
3C 078 & $1.09_{-0.48}^{+0.54}$ & $0.71 \pm 0.01$ & $0.84_{-0.16}^{+0.19}$ \\
3C 083 & $0.65_{-0.57}^{+2.10}$ & $0.83 \pm 0.01$ & $0.77_{-0.19}^{+0.72}$ \\
3C 84 & $1.18_{-0.18}^{+0.23}$ & $0.88 \pm 0.09$ & $0.98_{-0.01}^{+0.05}$ \\
3C 189 & $1.05_{-0.13}^{+0.33}$ & $0.75 \pm 0.01$ & $0.85_{-0.05}^{+0.11}$ \\
3C 270 & $0.82_{-0.56}^{+1.38}$ & $0.95 \pm 0.01$ & $0.90_{-0.19}^{+0.47}$ \\
3C 272.1 & $1.31_{-0.31}^{+0.63}$ & $0.69 \pm 0.01$ & $0.90_{-0.10}^{+0.22}$ \\
3C 274 & $1.18_{-0.07}^{+0.12}$ & $0.79 \pm 0.02$ & $0.93_{-0.02}^{+0.04}$ \\
3c 296 & $0.80_{-0.28}^{+0.46}$ & $0.86 \pm 0.04$ & $0.84_{-0.09}^{+0.16}$ \\
3C 317 & $0.97_{-0.13}^{+0.15}$ & $0.91 \pm 0.04$ & $0.93_{-0.03}^{+0.04}$ \\
3C 338 & $>1.08$ & $0.80 \pm 0.04$ & $>0.90$ \\
3C 346 & $0.97_{-0.13}^{+0.13}$ & $0.79 \pm 0.06$ & $0.85_{-0.02}^{+0.02}$ \\
3C 348 & $>0.68$ & $0.81 \pm 0.06$ & $>0.77$ \\
3C 438 & - & $>0.92$ & $>0.84$ \\
3C 449 & $>1.2$ & $0.66 \pm 0.02$ & $>0.86$ \\
\hline
\end{tabular}

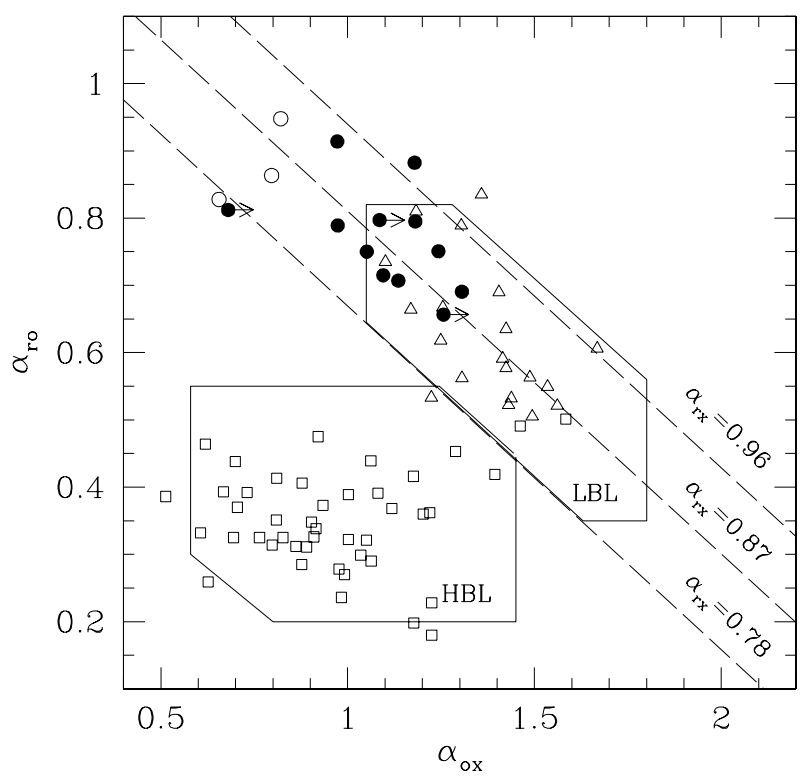

Fig. 4. Broad-band spectral indices, calculated with luminosity at $5 \mathrm{GHz}, 5500 \AA$ and $1 \mathrm{keV}$. The dashed lines represent constant values for the third index $\alpha_{\text {rx }}$. The solid lines indicate the regions of the plane within $2 \sigma$ from the mean $\alpha_{\text {ro }}$ and $\alpha_{\text {ox }}$ for BL Lacs drawn from the Deep X-Ray Radio Blazar Survey Padovani et al. (2003). Note that FR I cover approximately the same portion of the plane of LBL.

the sample but, as we argued in Sect. 3.2, this can be explained as due to the uncorrected dust absorption for the optical data.

We then compared these values with similar estimates for other classes of AGNs. Not surprisingly, radio quiet quasars have a median value of $\alpha_{\text {ro }}=-0.1$ and $\alpha_{\text {rx }}=0.36$ (Elvis et al. 1994), much lower than our radio loud sources, and this also applies to the Seyfert nuclei (Ho \& Peng 2001). But also the steep spectrum radio loud quasar have significantly lower values $\alpha_{\mathrm{ro}}=0.46$ and $\alpha_{\mathrm{rx}}=0.71$. These differences in spectral indices correspond to an optical (and X-ray) excess for a given radio core power by a factor between 20 and 40 .

Conversely, the FR I sources show a substantial overlap with the region of the plane populated by the Low energy peaked BL Lacs (LBL, Padovani \& Giommi 1995), see Fig. 4. For the comparison with BL Lacs we consider the radio-selected BL Lacs sample derived from the $1 \mathrm{Jy}$ catalogue (Stickel et al. 1991) and the BL Lac sample selected from the Einstein Slew survey (Elvis et al. 1992; Perlman et al. 1996). We used the classification into high and low energy peaked BL Lacs (HBL and LBL, respectively), as well as their multiwavelength data given by Fossati et al. (1998). The same result is obtained using BL Lac objects drawn from the Deep X-Ray Radio Blazar Survey by Padovani et al. (2003).

In BL Lacs the emission process is well established and the whole nuclear radiation is ascribed to the base of a relativistic jet. The similarity in the spectral index distributions of FR I and BL Lac provides further support of the idea that even in FR I the nuclear emission is non-thermal emission associated to an outflow.

A more detailed comparison with LBL is particularly instructive. The unified model for FR I and BL Lacs predicts that these two classes are drawn from the same population and are just seen at different angles with respect to the jet axis. In Fig. 5 we compare the spectral indices of the two groups also taking the extended radio-luminosity $L_{\text {ext }}$ into account. As an isotropic quantity, $L_{\text {ext }}$ does not depend on orientation and indeed LBL and FR I share the same range of $L_{\text {ext }}$. Thus FR I overlap in the spectral indices plane with the jet-dominated class with the "proper" level of radio-luminosity.

The differences between FR I and LBL, albeit quite small, warrant further attention to this point. In fact, FR I appear to be located only in the upper left corner characteristic of the LBL region (see Fig. 4). Figure 5 shows that this is not due to a mismatch of extended radio-luminosity between the two classes. However, as already discussed by Chiaberge et al. (2000) and Trussoni et al. (2003), Doppler beaming not only affects the angular pattern of the jet emission, but it also causes a shift in frequency that in turn modifies the observed spectral indices. It is clearly important to assess whether the observed (small) differences between the spectral indices are consistent with this idea.

In the top panel of Fig. 6, we give a simplified sketch to graphically illustrate the origin of this effect; we adopted a SED characteristic of a LBL (solid line), with the synchrotron peak in the FIR-mm region and a flat X-ray spectrum. The FR I SED (dotted line, re-normalized for clarity to have the same level of the synchrotron peak as the BL Lac) is shifted at lower energies and this produces a substantial decrease (relative to the radio) in the optical emission. Conversely the X-ray emission is (always relative to the radio) substantially unaltered. Indeed, no significant differences in $\alpha_{\mathrm{rx}}$ between FR I and LBL are present. Given the relatively large errors in the quantities involved in this comparison, and the possible effects of optical absorption described above, we prefer to refrain from a quantitative analysis. Nonetheless, this indicates that the small 

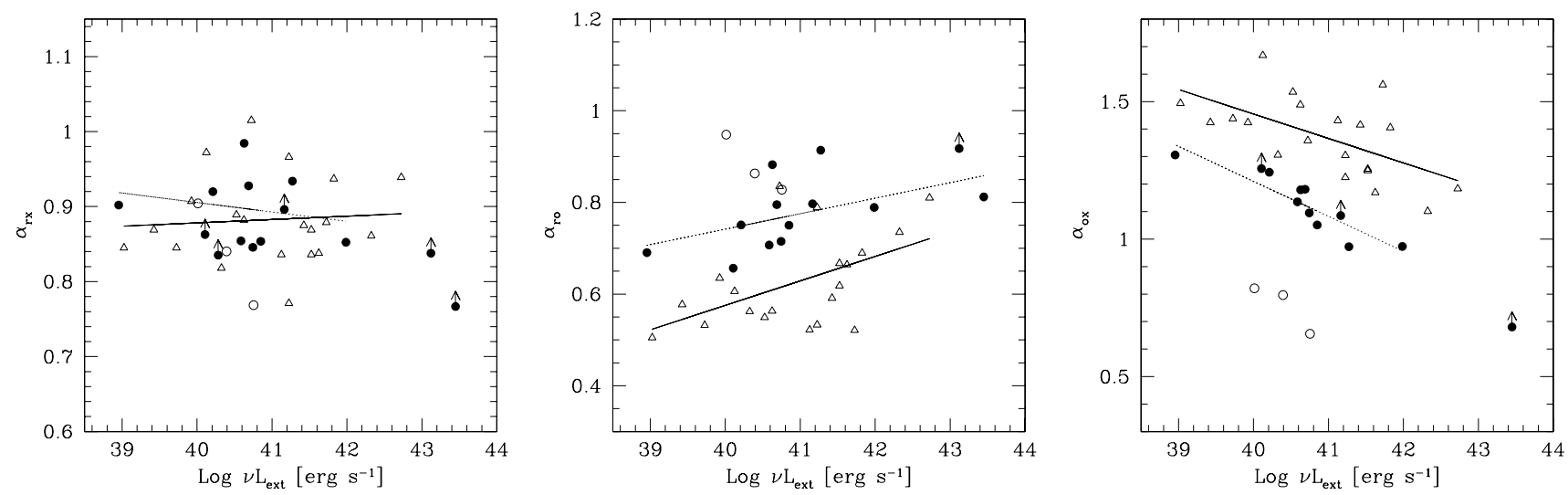

Fig. 5. Broad-band spectral indices plotted versus the extended radio luminosity at $178 \mathrm{MHz}$ for FR I (filled circles) and LBL (triangles). (Left) radio/X-ray, (middle) radio-optical, and (right) optical/X-ray. The empty circles represent the FR I sources for which we have detected a large $\left(N_{\mathrm{H}}>10^{22} \mathrm{~cm}^{-2}\right)$ intrinsic X-ray absorption. The solid and dotted lines represent the best linear fit for the changes in the spectral indices with $L_{\text {ext }}$ for FR I and LBL, respectively.

differences between the spectral indices of FR I and BL Lacs can be qualitatively accounted for by the presence of beaming.

\subsection{Spectral indices evolution with luminosity}

Figure 5 provides a further element in favour of a jet origin for the X-ray cores in FR I. Considering both FR I and LBL, a clear trend of the $\alpha_{\text {ro }}$ and $\alpha_{\text {ox }}$ spectral indices with radio luminosity is present: more powerful sources have higher $\alpha_{\text {ro }}$ (and lower $\alpha_{\mathrm{ox}}$ ) with respect to less luminous objects, while no trend between $\alpha_{\text {rx }}$ and $L_{\text {ext }}$ is apparent. FR I thus appear to follow the same evolution of spectral indices with the extended radio luminosity of LBL.

The slopes of the correlations between radio/optical and optical/X nuclear luminosity provide a different view of the same effect. In Sect. 3.2 we reported that the dependence of $L_{\mathrm{o}}$ on $L_{\mathrm{r}}$ is flatter than unity $(m=0.82 \pm 0.11)$, indicating an evolution with luminosity of the radio/optical ratio. The opposite behaviour is displayed by the $L_{\mathrm{X}} / L_{\mathrm{o}}$ relationship, which is steeper than unity $(m=1.16 \pm 0.09)$.

The dependence of the SED on luminosity over the whole class of blazar was recognized by Fossati et al. (1998) and is usually referred to as the "blazar sequence". Ghisellini et al. (1998) suggested that this is due to a relationship between the positions of the emission peaks with luminosity, related to the increasing cooling effects on the relativistic electrons. In particular, the synchrotron peak shifts at lower frequencies in the most powerful sources. We found evidence for a similar sequence among LBL, although it must be pointed out that we here consider the extended radio luminosity, while Fossati et al. (1998) used the radio-core luminosity. The optical emission, which in LBL is associated to the decreasing tail of the synchrotron component, would be increasingly reduced with respect to the radio when the source luminosity increases (see Fig. 6 for a sketch). This accounts for the trend in LBL observed in Fig. 5. A similar effect also seen in FR I suggests the presence of a similar luminosity sequence. This adds further weight to the similarity between BL Lacs and FR I and to a jet origin for the nuclei in radio-galaxies.

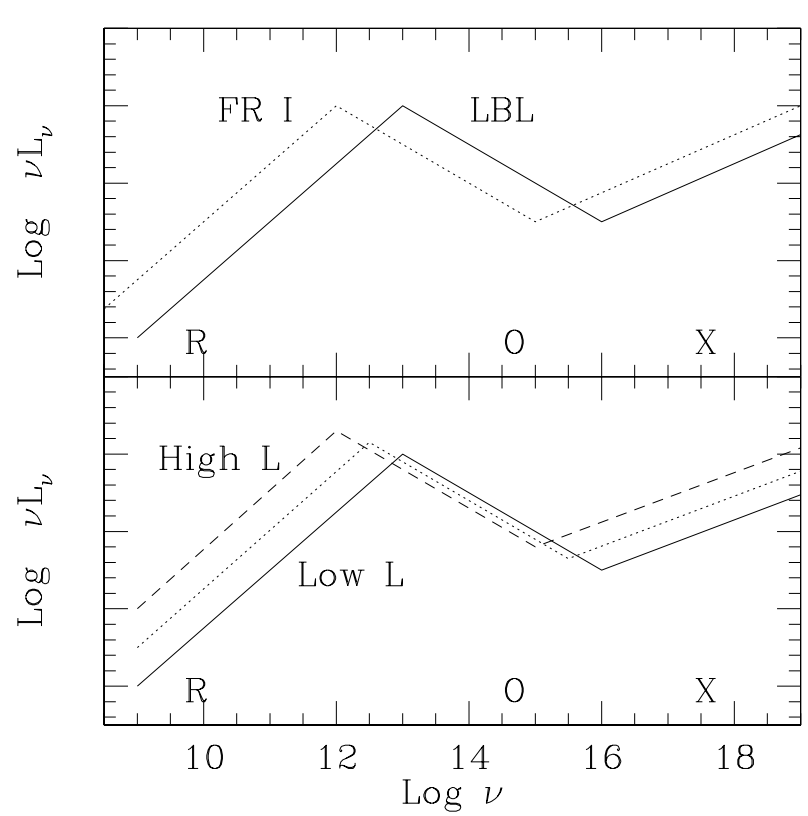

Fig. 6. Sketch of the SED evolution related to (top) frequency shift due to relativistic beaming and (bottom) a change in luminosity. See text for details.

\section{Summary and conclusions}

We presented results from Chandra observations of the 3C/FR I sample of low-luminosity radio-galaxies. Data are available for 18 out of the 33 sources of the sample, and we detected a power-law nuclear component, the characteristic signature of AGN emission, in 12 objects.

In 4 galaxies we detected nuclear $\mathrm{X}$-ray absorption at a level of $N_{\mathrm{H}} \sim(0.2-6) \times 10^{22} \mathrm{~cm}^{-2}$. The X-ray absorbed sources are associated with the presence of either highly inclined dusty disks seen in the HST images or to dust filaments directly projected onto the nuclei. Dust was also seen in most of the remaining sources, but no X-ray absorption was found with upper limits in the range $N_{\mathrm{H}} \leq 10^{21}-10^{22} \mathrm{~cm}^{-2}$. This suggests an association between dust (optical) and gas (X-ray) absorption but only when the geometry is favourable, supporting 
the existence of a flattened absorber, reminiscent of the standard thick tori envisaged by the AGN unified models. However, both the X-ray and (the indirect estimate of) the optical absorption, are substantially smaller when compared to what is typical of e.g. Seyfert galaxies. This indicates that we have an unobstructed view toward most FR I nuclei and that absorption plays only a marginal role in the remaining objects.

Concerning the properties of the obscuring medium, the ratio between optical and $\mathrm{X}$-ray absorption is in the range $A_{V} / N_{\mathrm{H}} \sim(0.7-3.3) \times 10^{-22}$ mag cm${ }^{2}$, slightly smaller than the standard galactic value, but not as extreme as found in other classes of AGN.

The most important issue is clearly the origin of the X-ray cores that can be produced either by the accretion process or by the associated outflow. Three results support this interpretation of a common origin for the radio, optical and X-ray cores, most likely associated to the base of a relativistic jet: i) the presence of strong correlations between the luminosities in the three bands, extending over 4 orders of magnitude and having a much smaller dispersion ( $~ 0.3$ dex) when compared to similar trends found for other classes of AGNs; ii) the close similarity of the broad-band spectral indices with the subclass of BL Lacs (for which the jet origin is well established) sharing the same range of extended radio-luminosity, in accordance with the FR I/BL Lacs unified model; iii) the presence of a common evolution of spectral indices with luminosity in both FR I and BL Lacs, most likely due to a shift in the synchrotron emission peak.

In the framework of a jet origin for the optical and X-ray cores, our analysis sets limits to the emission related to accretion at a fraction as small as $\sim 10^{-7}$ of the Eddington luminosity for a $10^{9} M_{\odot}$ black hole. Thus, regardless of the origin of the optical and X-ray nuclei, the luminosity of LLRG in the $\mathrm{X}$-ray band provides further evidence of a low level of accretion and/or for a low radiative efficiency of the accretion process. This was already noted by Chiaberge et al. (1999) from analysis of the optical images. The newly measured X-ray luminosities point to the same conclusion with the substantial advantage of being free from lingering doubts related to i) obscuration for the optical cores, since the X-ray spectra can be directly modeled accounting for the effects of absorption, and ii) the possibility that, due to a different SED behaviour with respect to brighter AGN, most of the nuclear emission is shifted at higher energies than the optical, as predicted by models of low efficiency accretion (e.g. Narayan et al. 2000; Di Matteo et al. 2000).

Acknowledgements. We thank Marco Chiaberge for useful comments and discussions during the preparation of the manuscript and the referee, Martin Hardcastle, for his comments and suggestions that improved this paper. This work was partly supported by the Italian MIUR under grant Cofin 2003/2003027534_002.

\section{References}

Auriemma, C., Perola, G. C., Ekers, R. D., et al. 1977, A\&A, 57, 41 Bennett, A. S. 1962, MmRAS, 68, 163
Bohlin, R. C., Savage, B. D., \& Drake, J. F. 1978, ApJ, 224, 132

Bower, G. A., Heckman, T. M., Wilson, A. S., \& Richstone, D. O. 1997, ApJ, 483, L33

Capetti, A., de Ruiter, H. R., Fanti, R., et al. 2000a, A\&A, 362, 871

Capetti, A., Trussoni, E., Celotti, A., Feretti, L., \& Chiaberge, M. 2000b, MNRAS, 318, 493

Capetti, A., Kleijn, G. V., \& Chiaberge, M. 2005, A\&A, 439, 935

Cardelli, J. A., Clayton, G. C., \& Mathis, J. S. 1989, ApJ, 345, 245

Chiaberge, M., Capetti, A., \& Celotti, A. 1999, A\&A, 349, 77

Chiaberge, M., Celotti, A., Capetti, A., \& Ghisellini, G. 2000, A\&A, 358,104

Chiaberge, M., Macchetto, F. D., Sparks, W. B., et al. 2002, ApJ, 571, 247

Colla, G., Fanti, C., Fanti, R., et al. 1975, A\&AS, 20, 1

Davis, J. E. 2001, ApJ, 562, 575

Di Matteo, T., Quataert, E., Allen, S. W., Narayan, R., \& Fabian, A. C. 2000, MNRAS, 311, 507

Donato, D., Sambruna, R. M., \& Gliozzi, M. 2004, ApJ, 617, 915

Edge, D. O., Shakeshaft, J. R., McAdam, W. B., Baldwin, J. E., \& Archer, S. 1959, MmRAS, 68, 37

Elvis, M., Plummer, D., Schachter, J., \& Fabbiano, G. 1992, ApJS, 80,257

Elvis, M., Wilkes, B. J., McDowell, J. C., et al. 1994, ApJS, 95, 1

Fabbiano, G. 1989, ARA\&A, 27, 87

Falcke, H., Malkan, M. A., \& Biermann, P. L. 1995, A\&A, 298, 375

Fanaroff, B. L., \& Riley, J. M. 1974, MNRAS, 167, 31

Fossati, G., Maraschi, L., Celotti, A., Comastri, A., \& Ghisellini, G. 1998, MNRAS, 299, 433

Ghisellini, G., Celotti, A., Fossati, G., Maraschi, L., \& Comastri, A. 1998, MNRAS, 301, 451

Hardcastle, M. J., \& Worrall, D. M. 2000, MNRAS, 314, 359

Hardcastle, M. J., Worrall, D. M., Birkinshaw, M., \& Canosa, C. M. 2003, MNRAS, 338, 176

Ho, L. C., \& Peng, C. Y. 2001, ApJ, 555, 650

Huchra, J., \& Burg, R. 1992, ApJ, 393, 90

Isobe, T., Feigelson, E. D., Akritas, M. G., \& Babu, G. J. 1990, ApJ, 364, 104

Jaffe, W., Ford, H., Ferrarese, L., van den Bosch, F., \& O'Connell, R. W. 1996, ApJ, 460, 214

Kormendy, J., \& Richstone, D. 1995, ARA\&A, 33, 581

Maiolino, R., Marconi, A., Salvati, M., et al. 2001, A\&A, 365, 28

Merloni, A., Heinz, S., \& di Matteo, T. 2003, MNRAS, 345, 1057

Narayan, R., Igumenshchev, I. V., \& Abramowicz, M. A. 2000, ApJ, 539, 798

Osterbrock, D. E., \& Martel, A. 1993, ApJ, 414, 552

Padovani, P., \& Giommi, P. 1995, ApJ, 444, 567

Padovani, P., Perlman, E. S., Landt, H., Giommi, P., \& Perri, M. 2003, ApJ, 588, 128

Perlman, E. S., Stocke, J. T., Schachter, J. F., et al. 1996, ApJS, 104, 251

Risaliti, G., Maiolino, R., \& Salvati, M. 1999, ApJ, 522, 157

Sadler, E. M., Jenkins, C. R., \& Kotanyi, C. G. 1989, MNRAS, 240, 591

Schmitt, J. H. M. M. 1985, ApJ, 293, 178

Sparks, W. B., Baum, S. A., Biretta, J., Macchetto, F. D., \& Martel, A. R. 2000, ApJ, 542, 667

Stickel, M., Fried, J. W., Kuehr, H., Padovani, P., \& Urry, C. M. 1991, ApJ, 374, 431

Trussoni, E., Capetti, A., Celotti, A., Chiaberge, M., \& Feretti, L. 2003, A\&A, 403, 889

Worrall, D. M., \& Birkinshaw, M. 1994, ApJ, 427, 134 